\title{
Collaborative care speeds recovery from depression
}

Hedrick SC, Chaney EF, Felker B et al. Effectiveness of collaborative care depression treatment in Veterans' Affairs primary care.J Gen Int Med 2003;18:9-16.

\section{QUESTION: Does collaborative care compared with consult-liaison care improve depressive symptoms in people with major depression or dysthymia?}

\section{Design}

Randomised controlled trial.

\section{Setting}

Veterans' Affairs primary care clinic, Seattle, USA.

\section{Participants}

354 people with major depression, dysthymia or both (Primary Care Evaluation of Mental Disorders and DSM-IV criteria). Main exclusion criteria were included being treated by a specialist or being treated for risk of suicide, acute psychosis, post-traumatic stress disorder or substance abuse.

\section{Intervention}

Collaborative team-led, guideline-based treatment plan with monitoring of the plan's implementation in primary care, and patient support versus traditional psychiatric specialist consultation and liaison. The collaborative specialist team, who developed treatment plans based on published guidelines, included a clinical psychologist, psychiatrist, social workers and psychology technician. People in the control group had access to a psychologist and social workers to ensure that both groups were similarly resourced.

\section{Main outcome measures}

Depressive symptoms (Hopkins Symptom Checklist [SCL-20]; health and mental health status (Veterans Short Form [SF]-36 Health Status Questionnaire); effects on work and social life (Sheehan Disability Scale) and number and dosage of antidepressant prescriptions.

\begin{abstract}
Main results
Collaborative care significantly reduced depressive symptoms and interference with work and social life at 3-month, but not 9-month follow up (see table). At 9 months, collaborative care significantly improved mental health status and increased the number of antidepressant prescriptions compared with traditional care (45\% v 36\% with mental health status improvement, $\mathrm{p}=0.03 ; 15 \%$ v $25 \%$ with mental health status decline, $\mathrm{p}=0.03 ; 80 \%$ v $62 \%$ receiving prescriptions, $\mathrm{p}<0.001$ ).
\end{abstract}

\section{Conclusions}

Collaborative care speeds recovery and leads to sustained improvements in mental health.

Sources of funding:
The Department of
Veterans Affairs,
Veterans Health
Administration, Health
Services Research and
Development Service.
Correspondence to:
Dr Hedrick, HSREDD
(152) VA Puget Sound
Health Care System,
1660, S. Columbian
Way, Seattle, WA
98108.email:
susan.hedrick@
med.va.gov

Table: Mean difference in scores between collaborative care and consult liaison, adjusted for baseline score $(95 \%$ CI)

\begin{tabular}{lll} 
& 3 months $(95 \% \mathrm{CI})$ & 9 months $(95 \% \mathrm{CI})$ \\
$\begin{array}{l}\text { Hopkins Symptom } \\
\text { Checklist }\end{array}$ & $-0.17(-0.31$ to -0.03$)$ & $-0.13(-0.29$ to +0.03$)$ \\
\hline Sheehan Disability Scale & $-0.53(-1.04$ to -0.02$)$ & $-0.43(-0.99$ to +0.13$)$ \\
\hline
\end{tabular}

\section{COMMENTARY}

The main take away message for clinicians from this study is that systematic care planning and monitoring of depressed patients in primary care will result in more use of drug and psychotherapy and moderate improvements in short term outcome. It is encouraging that a less intensive intervention with a more disadvantaged and ill VA population continues to demonstrate the benefits shown in previous trials of shared care in the community.

The disappointing 9-month outcomes raise questions about the adequacy of the maintenance phase of treatment. Only $60 \%$ of the patients in both treatment groups received guideline care for 90 days and there were high rates (40\%) of no shows and cancellations of outpatient visits. The phone support that was planned to occur in the maintenance phase was poorly implemented (only one out of the three to five planned calls were completed). Since other studies $^{1}$ have shown that continuation of therapy influences outcome, future interventions need to strengthen efforts to achieve adherence beyond the acute care period.

Policy makers will be interested in the cost effectiveness analysis of this study published subsequent to this report. ${ }^{2}$ In the current report the authors emphasise the importance of attending to financial incentives when implementing shared care resources at a systems level. This conclusion holds across different health care systems $\mathrm{s}^{3-4}$ and is a critical ingredient to the widespread adoption of effective collaborative relationships. There is a renewed focus upon the reform of primary care many jurisdictions. Evidence about the effectiveness of linking specialty mental health care providers into family practice is timely and has the potential to improve health outcomes for large numbers of depressed patients.

Dr Paula Goering, RN PhD Director, Health Systems Research and Consulting Unit Department of Psychiatry and Faculty of Nursing Centre for Addiction and Mental Health University of Toronto, Canada

1 Melfi CA, Chawla AJ, Croghan TW, Hanna MP, Kennedy S, Sredl, K. The effects of adherence to antidepressant treatment guidelines on relapse and recurrence of depression. Arch Gen Psychiatry 1998; 55: 1128-1132.

2 Liu C-F, Hedrick SC, Chaney EF et al. Cost-effectiveness of collaborative care for depression in a primary care veteran population. Psychiatr Serv 2003 , 54: $698-704$. Dewa CS, Hoch JS, Goering P. Using financial incentives to promote shared mental health care. Can J Psych 2001; 46: 488-495.

Frank RG, Huskamp HA, Pincus HA. Aligning incentives in the treatment of depression in primary care with evidence-based practice. Psychiatr Serv 2003; 54: $682-687$. 